\title{
Association between serum calcium levels and prognosis, hematoma volume, and onset of cerebral hemorrhage in patients undergoing hemodialysis
}

Mineaki Kitamura ${ }^{1,2^{*}}$ D, Yohei Tateishi ${ }^{3}$, Shuntaro Sato ${ }^{4}$, Satoko Kitamura ${ }^{1,2}$, Yuki Ota $^{2}$, Kumiko Muta ${ }^{2}$, Hiroshi Yamashita ${ }^{2}$, Tadashi Uramatsu ${ }^{2}$, Yoko Obata ${ }^{2}$, Yasushi Mochizuki ${ }^{1}$, Masaharu Nishikido ${ }^{5}$, Tsuyoshi Izumo ${ }^{6}$, Takashi Harada ${ }^{7}$, Satoshi Funakoshi ${ }^{7}$, Takayuki Matsuo ${ }^{6}$, Akira Tsujino ${ }^{3}$, Hideki Sakai ${ }^{1}$, Hiroshi Mukae ${ }^{8}$ and Tomoya Nishino ${ }^{2}$

\begin{abstract}
Background: High serum calcium levels should be avoided in patients on hemodialysis (HD) because they can induce cardiovascular diseases and worsen the patient's prognosis. In contrast, low serum calcium levels worsen the prognosis of patients with cerebral hemorrhage in the general population. So far, whether serum calcium levels in patients on HD are associated with cerebral hemorrhage remains unknown. This study aimed to reveal the association between serum calcium and cerebral hemorrhage in patients on HD, including in-hospital death, volume of hematoma, and onset of cerebral hemorrhage.

Methods: This cross-sectional case-control study included 99 patients on HD with cerebral hemorrhage at a single center between July 1, 2007 and December 31, 2017. Controls included 339 patients on HD at a single HD center between July 1, 2011 and June 30, 2012. Data on serum calcium level, patient demographics, and comorbid conditions were collected, and associations between cerebral hemorrhage and subsequent death were evaluated by multivariate logistic regression analysis. Further, the association of these backgrounds and hematoma volume was evaluated by multiple regression analysis.
\end{abstract}

Results: Of the 99 patients, 32 (32\%) died from cerebral hemorrhage. The corrected serum calcium level (odds ratio $[\mathrm{OR}], 2.49 ; 95 \%$ confidence interval $[\mathrm{Cl}], 1.43-4.35 ; P<0.001)$ and antiplatelet drug use $(\mathrm{OR}, 3.95 ; 95 \% \mathrm{Cl}, 1.50-10.4$; $P=0.005)$ had significant effects on the prognosis. Moreover, the corrected serum calcium $(P=0.003)$ and antiplatelet drug use $(P=0.01)$ were significantly correlated with hematoma volume. In the patients, the corrected serum calcium level $(\mathrm{OR}, 1.54 ; 95 \% \mathrm{Cl}, 1.07-2.22 ; P=0.02)$ was associated with the onset of cerebral hemorrhage, as was pre-hemodialysis systolic blood pressure (per $10 \mathrm{mmHg})(\mathrm{OR}, 1.40 ; 95 \% \mathrm{Cl}, 1.23-1.59$; $\mathrm{P}<0.001)$.

Conclusions: Although the precise mechanisms remain unknown, a high serum calcium level is associated with cerebral hemorrhage in patients on HD. Thus, we should pay attentions to a patient's calcium level.

Keywords: Cerebral hemorrhage, Hemodialysis, Serum calcium

\footnotetext{
* Correspondence: minekitamura@nagasaki-u.ac.jp

${ }^{1}$ Division of Blood Purification, Nagasaki University Hospital, Nagasaki, Japan

${ }^{2}$ Department of Nephrology, Nagasaki University Hospital, Nagasaki, Japan

Full list of author information is available at the end of the article
}

(c) The Author(s). 2019 Open Access This article is distributed under the terms of the Creative Commons Attribution 4.0 International License (http://creativecommons.org/licenses/by/4.0/), which permits unrestricted use, distribution, and reproduction in any medium, provided you give appropriate credit to the original author(s) and the source, provide a link to the Creative Commons license, and indicate if changes were made. The Creative Commons Public Domain Dedication waiver (http://creativecommons.org/publicdomain/zero/1.0/) applies to the data made available in this article, unless otherwise stated. 


\section{Background}

Stroke is a major cause of death and the leading cause of disability in patients on hemodialysis (HD) worldwide, where its incidence is several times higher than in the general population [1-6]. Although the recent incidence of cerebral infarction was found to be higher than that of cerebral hemorrhage in patients on HD [7], cerebral hemorrhage had a greater impact on patients' prognoses compared with cerebral infarction $[4,6,8]$. To prevent cerebral hemorrhage or mitigate the severity of cerebral hemorrhage, modifiable factors in patients on HD are crucial.

Several traditional risk factors for cerebral hemorrhage in patients on HD have been elucidated, such as male sex [9], absence of antihypertensive drug [10], predialysis hypertension $[2,3,5,7,9]$, low $\mathrm{KT} / \mathrm{V}$ [3], and high blood hemoglobin [11]. In addition to chronic kidney disease (CKD)-mineral bone disorder, a high serum phosphate level [9] and high intact parathyroid hormone (iPTH) level [12] are known risk factors for cerebral hemorrhage in patients on HD.

Since multiple studies have reported that a high calcium concentration is associated with cardiovascular diseases and a poor prognosis in patients with CKD [13-15], the KDIGO guidelines have changed the recommendations for avoiding hypercalcemia in patients on HD, although the treatment range of serum calcium has been left unchanged $[16,17]$.

In contrast, studies that were not restricted to patients with end-stage renal disease (ESRD) have demonstrated that a low serum calcium level worsens patients' prognoses and enlarges the hematoma volume in acute cerebral hemorrhage [18-20].

We decided to investigate the contradicting content of whether serum calcium levels in HD patients with cerebral hemorrhage affect patients' prognosis or onset. This study aimed to reveal the association between serum calcium and cerebral hemorrhage in patients on HD, including in-hospital death, volume of hematoma, and onset of cerebral hemorrhage.

\section{Methods}

\section{Study design}

This is a cross-sectional case-control study in the same medical zone. The cases were of patients on HD with cerebral hemorrhage who were admitted to Nagasaki University Hospital or of patients on HD who developed cerebral hemorrhage at Nagasaki University Hospital during their stay for other treatments, but patients with traumatic hemorrhage, subarachnoid hemorrhage, and hemorrhage after ischemic stroke were excluded. The study period was between July 1, 2007, and December 31, 2017. Patients were diagnosed with cerebral hemorrhage using computed tomography (CT) at Nagasaki University Hospital within 3 days of experiencing neurological symptoms.
Cases of traumatic hemorrhage were excluded based on the decision of the neurosurgeons of our hospital and imaging diagnosis at admission. Treatment strategies were discussed by multiple neurosurgeons and neurologists. The controls included patients who underwent HD between July 1, 2011, and June 30, 2012, in Nagasaki Renal Center, which is the largest HD facility in Nagasaki City. Almost a quarter of patients on HD at Nagasaki City medical zone were enrolled. Patients who did not undergo HD in their birth month were excluded because data collected in the birth month and on birthdays were considered substituted events.

\section{Data collection}

Baseline characteristics of patients were collected through a retrospective review of hospital medical records and medical referral letters. In addition, data on the HD status just before admission, activities of daily living (ADL) before onset, and medications as well as laboratory data obtained closest to the event were collected from the medical referral letters. The hematoma volume was determined from a CT scan on admission or just after onset by a simplified formula as follows: maximum transverse diameter $\times$ maximal anteroposterior diameter $\times$ maximal superoinferior diameter $\times 1 / 2[3,21]$, which was natural-log transformed as in a previous study [22]. The onset of time was estimated from medical referral letters; if it was impossible, we assigned noon as the time of onset, as in previous reports [23]. The Glasgow Coma Scale (GCS) score, National Institutes of Health Stroke Scale (NIHSS) score [24], and severity of cerebral hemorrhage were defined by ADL at discharge, which was evaluated by the modified Rankin scale (mRS) [25], as follows: 0 , no symptoms at all; 1 , no significant disability despite symptoms; 2 , slight disability; 3 , moderate disability (able to walk without assistance); 4, moderately severe disability; 5 , severe disability (bed ridden); and 6, death.

For the controls (control group), data were collected from the medical records of Nagasaki Renal Center. Similar to that in patients with cerebral hemorrhage (cerebral hemorrhage group), data were collected in the period before the patient's birthday. The corrected serum calcium levels were obtained using the Payne equation [26]. A ratio of 1:200 was applied to convert the darbepoetin-alfa and epoetin-beta pegol doses to their equivalent epoetin doses [27].

\section{Statistical analysis}

Categorical variables were expressed as number (\%), whereas continuous values were expressed as mean \pm standard deviations (SD). Non-normally distributed data were presented as median values with interquartile ranges. Statistical analyses were performed using JMP 13 software (SAS Institute Inc., NC, USA). Wilcoxon rank sum test and the chi-squared test were used to evaluate 
differences between groups. Patients with cerebral hemorrhage were divided into four groups (Q1-Q4) with the cutoff values being the 25th, 50th, and 75th percentiles of the corrected serum calcium levels. Analysis of variance and Cochran-Armitage test were used to demonstrate the trend. Univariate and multivariate logistic regression analyses were also performed.

In the multivariate logistic regression analyses for the prognosis of cerebral hemorrhage patients, model 1 was only adjusted for age and sex, and parameters of model 2 were determined using a stepwise model (mixed method) with an inclusion criterion of $P<0.1$ in univariate logistic regression analysis in addition to the corrected serum calcium level. In the comparison of the cerebral hemorrhage group and control group, model 1 adopted the risk factors of traditional cerebral hemorrhage, including dialysis vintage, history of diabetes, systolic blood pressure at initiation of HD proximal to onset, hemoglobin, phosphate, iPTH, and corrected serum-calcium. In model 2, parameters were determined using a stepwise model (mixed method) as described above.

Multiple regression analysis was performed to predict hematoma volume. The constitutional parameters were age, sex, and those associated with hematoma volume, such as systolic blood pressure (sBP) pre-HD [3] and corrected serum calcium levels $[18,20]$, and residual parameters were selected using the stepwise method (mixed method). An inclusion criterion of $\mathrm{P}<0.1$ in the

\section{a Patients with cerebral hemorrhage}

Patients with cerebral hemorrhage on hemodialysis Confirmed by Computed Tomography $(\mathrm{N}=154)$

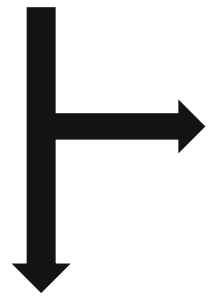

$$
\text { Excluded }(\mathrm{N}=55)
$$

-Traumatic hemorrhage $(\mathrm{N}=43)$

Subarachnoid Hemorrhage $(\mathrm{N}=6)$

Hemorrhagic cerebral infarction $(\mathrm{N}=4)$ -Incomplete data $(\mathrm{N}=2)$

Included ( $\mathrm{N}=99)$

Patients with cerebral hemorrhage on hemodialysis

\section{b Control Patients}

$$
\begin{aligned}
& \text { Patients on hemodialysis in the Nagasaki Renal Center } \\
& \text { between July 1, } 2011 \text { and June 30, } 2012(\mathrm{~N}=402)
\end{aligned}
$$

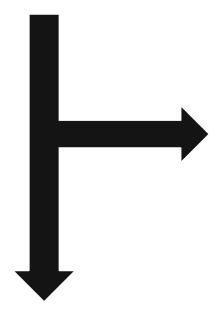

Excluded $(\mathrm{N}=63)$

-Death or relocation to other facilities prior to their birth month $(\mathrm{N}=30)$

- Moving into the Nagasaki Renal Center

after their birth month $(\mathrm{N}=33)$

Included ( $\mathrm{N}=339)$

As control patients on hemodialysis

Fig. 1 Patient flow diagram. a Patients with cerebral hemorrhage in Nagasaki University Hospital. b Control patients in the Nagasaki Renal Center between July 1, 2011 and June 2012 
univariate logistic regression analysis was used. Adjusted odds ratios (ORs) and 95\% confidence intervals (CIs) were calculated. A $P$ value $<0.05$ was considered statistically significant.

Past histories were excluded as constitutional parameters in comparisons of the cases and controls because of unavoidable information bias due to the differences in data collection methods. Missing data were removed from the analyses, and the remaining data were used.

\section{Ethical consideration}

All procedures involving human participants were performed in accordance with the ethical standards of the institutional review board (IRB) of Nagasaki University Hospital (1602221-2) and Nagasaki Renal Center (Nagasaki, Japan) (30001) and of the 1964 Declaration of Helsinki and its later amendments. Although all patients in this study were informed of the investigations being performed, the study was a medical record-based retrospective analysis and the included patients were anonymized. Therefore, the IRB approved the exemption from obtaining written informed consent.

\section{Results}

Demographic data for patients with cerebral hemorrhage The participants included 96 HD patients with cerebral hemorrhage who were admitted to Nagasaki University Hospital between 2007 and 2017 and 3 HD patients who developed cerebral hemorrhage in the hospital. The patient flow diagram is shown in Fig. 1. The severity of hemorrhage in terms of GCS, NIHSS, hematoma volume at admission, and mRS at discharge is shown in Table 1. The average age was $64.8 \pm 10.7$ years (men, 66; women, 33 ), and the median dialysis vintage was 87 months (Table 2). The median date of the onset of cerebral hemorrhage was May 21, 2012. Figure 2a shows hemorrhagic areas, and
Fig. $2 \mathrm{~b}$ shows the distribution of the hematoma volume. Severe outcomes were observed in 66 patients (67\%): 32 died (mRS: 6) and 34 were severely disabled (mRS: 5). Figure 2c shows the total $\mathrm{mRS}$ score at discharge.

Significant differences were observed in the GCS, NIHSS, intraventricular hemorrhage, and volume of hematoma between patients who died and those who survived (Table 1).

\section{Characteristics of HD patients with cerebral hemorrhage and controls}

Patients with cerebral hemorrhage and controls were compared to clarify the correlations for the onset of cerebral hemorrhage. The number of patients who underwent HD in the Nagasaki Renal Center between July 1, 2011, and June 30, 2012, was 402; however, 30 were excluded because of death or relocation to other facilities before their birth month, and 33 were excluded because of moving to our facility after their birth month. Therefore, the number of control patients on HD was 339 (Fig. 1). Table 2 (right side) shows the demographic data of patients with cerebral hemorrhage and the controls.

\section{Differences between patients who died and those who survived following cerebral hemorrhage}

Table 2 (left side) shows patients' background data, which were confirmed or examined during the precritical stage, and the differences between patients who died and those who survived. Significant differences were observed in the past histories of ischemic heart disease and heart valve repair or replacement, use of antiplatelet drugs and warfarin, and corrected serum calcium levels.

According to a multivariate logistic analysis, corrected serum calcium, antiplatelet use, and heart valve repair or

Table 1 Cerebral hemorrhage patients' condition on admission

\begin{tabular}{|c|c|c|c|c|}
\hline & Total $(N=99)$ & Died $(N=32)$ & Survived $(N=67)$ & $P$ value \\
\hline \multicolumn{5}{|l|}{ Features of cerebral hemorrhage } \\
\hline sBP on admission (mmHg) & $184 \pm 34$ & $187 \pm 36$ & $183 \pm 33$ & 0.7 \\
\hline dBP on admission (mmHg) & $96 \pm 23$ & $100 \pm 25$ & $94 \pm 22$ & 0.3 \\
\hline Pulse pressure on admission $(\mathrm{mmHg})$ & $80 \pm 18$ & $81 \pm 24$ & $80 \pm 15$ & 0.9 \\
\hline Glasgow Coma Scale & $10 \pm 4$ & $6 \pm 3$ & $12 \pm 4$ & $<0.001$ \\
\hline NIHSS & $15 \pm 10$ & $23 \pm 9$ & $11 \pm 9$ & $<0.001$ \\
\hline Intraventricular hemorrhage (\%) & 64 & 97 & 48 & $<0.001$ \\
\hline Volume of hematoma $(\mathrm{mL})$ & $50 \pm 60$ & $103 \pm 70$ & $24 \pm 33$ & $<0.001$ \\
\hline Surgical hematoma evacuation (\%) & 31 & 34 & 30 & 0.7 \\
\hline Onset during 2-day interval HD (\%) & 37 & 41 & 36 & 0.6 \\
\hline Time for patients to transport (hour) ${ }^{a}$ & $4(3-12)$ & $6(3-12)$ & $4(3-11)$ & 0.4 \\
\hline
\end{tabular}

$d B P$ diastolic blood pressure, $H D$ hemodialysis, NIHSS National Institute of Health Stroke Scale, sBP systolic blood pressure Continuous variables are shown as mean \pm standard deviation and categorical variables, as percentage or number (percentage)

${ }^{a}$ Median (interquartile range) 
Table 2 Demographic data of patients with cerebral hemorrhage divided by dead or alive and control patients

\begin{tabular}{|c|c|c|c|c|c|c|}
\hline & Died $(N=32)$ & Survived $(N=67)$ & $P$ value & $\mathrm{CH}$ all $(\mathrm{N}=99)$ & Control $(N=339)$ & $P$ value \\
\hline Age (year) & $64.9 \pm 9.2$ & $64.7 \pm 11.5$ & 0.7 & $64.8 \pm 10.7$ & $67.4 \pm 13.3$ & 0.06 \\
\hline Male (\%) & 62.5 & 68.7 & 0.5 & 66.7 & 57.2 & 0.09 \\
\hline HD vintage (months) ${ }^{a}$ & $85(30-169)$ & $88(27-177)$ & 0.7 & $87(29-177)$ & $56(23-122)$ & 0.04 \\
\hline \multicolumn{7}{|l|}{ Cause of ESRD } \\
\hline Chronic glomerulonephritis (\%) & 28 & 48 & & 41 & 38 & \\
\hline Diabetes (\%) & 44 & 31 & & 35 & 28 & \\
\hline Nephrosclerosis (\%) & 13 & 7 & & 9 & 17 & \\
\hline ADPKD (\%) & 6 & 7 & & 7 & 4 & \\
\hline Others (\%) & 6 & 6 & & 6 & 6 & \\
\hline Unknown (\%) & 3 & 0 & 0.3 & 1 & 7 & 0.02 \\
\hline \multicolumn{7}{|l|}{ Past history } \\
\hline Diabetes mellitus (\%) & 44 & 37 & 0.5 & 39 & 35 & 0.4 \\
\hline Ischemic heart disease (\%) & 34 & 9 & 0.002 & 17 & 34 & $<0.001$ \\
\hline Atrial fibrillation (\%) & 3 & 12 & 0.11 & 9 & 10 & 0.8 \\
\hline Heart valve repair or replacement (\%) & 16 & 2 & 0.008 & 6 & 4 & 0.2 \\
\hline History of cerebral infarction (\%) & 31 & 34 & 0.8 & 33 & 25 & 0.09 \\
\hline History of cerebral hemorrhage (\%) & 16 & 13 & 0.8 & 14 & 6 & 0.02 \\
\hline \multicolumn{7}{|l|}{ Medication } \\
\hline Number of antihypertensive drugs & $1.6 \pm 1.2$ & $1.9 \pm 1.3$ & 0.3 & $1.8 \pm 1.3$ & $1.6 \pm 1.5$ & 0.09 \\
\hline Antiplatelet use (\%) & 65 & 36 & 0.008 & 45 & 39 & 0.3 \\
\hline Warfarin use (\%) & 25 & 7 & 0.02 & 13 & 7 & 0.07 \\
\hline Statin use (\%) & 17 & 9 & 0.3 & 11 & 16 & 0.3 \\
\hline Vitamin D use (\%) & 54 & 66 & 0.3 & 62 & 67 & 0.1 \\
\hline Phosphate binder use (\%) & 71 & 68 & 0.8 & 69 & 65 & 0.5 \\
\hline $\mathrm{CaCO}_{3}$ use (\%) & 52 & 47 & 0.7 & 48 & 48 & 1.0 \\
\hline Cinacalcet use (\%) & 13 & 18 & 0.6 & 16 & 17 & 0.9 \\
\hline Walking independently (mRS $\leqq 3)(\%)$ & 77 & 86 & 0.2 & 83 & 80 & 0.5 \\
\hline \multicolumn{7}{|l|}{ Dialysis conditions } \\
\hline Dialyzing time $(\mathrm{hr})^{\mathrm{a}}$ & $4(3-4)$ & $4(3.5-4)$ & 0.7 & $4(3.5-4)$ & $4(3-4)$ & 0.02 \\
\hline Dry weight (kg) & $52.4 \pm 11.5$ & $53.7 \pm 11.5$ & 0.4 & $53.3 \pm 11.5$ & $52.1 \pm 11.0$ & 0.3 \\
\hline Fluid removal per dry weight proximate to the onset (\%) & $3.14 \pm 2.04$ & $3.35 \pm 1.71$ & 0.6 & $3.29 \pm 1.81$ & $3.10 \pm 1.74$ & 0.3 \\
\hline Dialysate Ca concentration (mEq/L) & $2.81 \pm 0.14$ & $2.79 \pm 0.18$ & 0.7 & $2.79 \pm 0.17$ & 2.75 & NA \\
\hline Online HDF (\%) & 13 & 7 & 0.4 & 9 & 22 & 0.002 \\
\hline sBP pre-HD proximate to the onset $(\mathrm{mmHg})$ & $167 \pm 31$ & $167 \pm 22$ & 1.0 & $167 \pm 25$ & $150 \pm 24$ & $<0.001$ \\
\hline $\mathrm{dBP}$ pre-HD proximate to the onset $(\mathrm{mmHg})$ & $86 \pm 14$ & $87 \pm 16$ & 0.8 & $87 \pm 16$ & $78 \pm 13$ & $<0.001$ \\
\hline Pulse pressure proximate to the onset $(\mathrm{mmHg})$ & $81 \pm 24$ & $80 \pm 15$ & 1.0 & $80 \pm 18$ & $72 \pm 21$ & $<0.001$ \\
\hline ESA (U/week) & $6719 \pm 3592$ & $6296 \pm 4556$ & 0.6 & $6435 \pm 4247$ & $5513 \pm 4396$ & 0.03 \\
\hline Epoetin (\%) & 38 & 28 & & 31 & 9 & \\
\hline Darbepoetin (\%) & 41 & 37 & & 38 & 47 & \\
\hline CERA (\%) & 13 & 18 & & 16 & 27 & \\
\hline Intravenous iron use (\%) & 22 & 27 & 0.6 & 25 & 19 & 0.08 \\
\hline \multicolumn{7}{|l|}{ Laboratory parameters } \\
\hline $\mathrm{Hb}(\mathrm{g} / \mathrm{dL})$ & $10.6 \pm 1.9$ & $10.6 \pm 1.5$ & 0.4 & $10.6 \pm 1.7$ & $10.8 \pm 1.4$ & 0.09 \\
\hline Ferritin $(\mathrm{ng} / \mathrm{mL})^{\mathrm{a}}$ & $139(54-284)$ & $71(31-167)$ & 0.095 & $85(34-212)$ & $53(21-154)$ & 0.03 \\
\hline
\end{tabular}


Table 2 Demographic data of patients with cerebral hemorrhage divided by dead or alive and control patients (Continued)

\begin{tabular}{|c|c|c|c|c|c|c|}
\hline & Died $(N=32)$ & Survived $(N=67)$ & $P$ value & $\mathrm{CH}$ all $(\mathrm{N}=99)$ & Control $(N=339)$ & $P$ value \\
\hline TSAT (\%) & $21.3 \pm 11.3$ & $24.4 \pm 12.9$ & 0.4 & $23.4 \pm 12.4$ & $24.3 \pm 13.0$ & 0.6 \\
\hline BUN (mg/dL) & $61.4 \pm 20.7$ & $60.2 \pm 14.9$ & 0.8 & $60.6 \pm 16.8$ & $68.1 \pm 18.2$ & $<0.001$ \\
\hline Creatinine (mg/dL) & $9.1 \pm 3.2$ & $10.0 \pm 3.1$ & 0.4 & $9.8 \pm 3.1$ & $10.2 \pm 3.4$ & 0.2 \\
\hline Corrected serum calcium (mg/dL) & $9.93 \pm 0.91$ & $9.27 \pm 0.86$ & 0.001 & $9.48 \pm 0.93$ & $9.22 \pm 0.92$ & 0.01 \\
\hline Phosphate (mg/dL) & $5.47 \pm 2.09$ & $4.93 \pm 1.47$ & 0.3 & $5.09 \pm 1.68$ & $5.58 \pm 1.57$ & 0.007 \\
\hline iPTH $(\mathrm{pg} / \mathrm{mL})^{\mathrm{a}}$ & $122(45-319)$ & $124(45-202)$ & 0.7 & $124(45-202)$ & $72(28-155)$ & 0.007 \\
\hline ALP (IU/L) & $305 \pm 103$ & $285 \pm 143$ & 0.14 & $291 \pm 131$ & $282 \pm 133$ & 0.4 \\
\hline Serum albumin (g/dL) & $3.41 \pm 0.56$ & $3.62 \pm 0.50$ & 0.07 & $3.55 \pm 0.53$ & $3.56 \pm 0.43$ & 0.9 \\
\hline $\mathrm{CRP}(\mathrm{mg} / \mathrm{dL})^{\mathrm{a}}$ & $0.39(0.09-0.69)$ & $0.17(0.10-0.61)$ & 0.3 & $0.20(0.09-0.65)$ & $0.18(0.07-0.53)$ & 0.2 \\
\hline T-Chol (mg/dL) & $152 \pm 35$ & $156 \pm 38$ & 0.9 & $155 \pm 37$ & $162 \pm 37$ & 0.07 \\
\hline Triglyceride (mg/dL) & $102 \pm 60$ & $89 \pm 44$ & 0.5 & $93 \pm 49$ & $106 \pm 64$ & 0.2 \\
\hline
\end{tabular}

$A D P K D$ autosomal dominant polycystic kidney disease, $A L P$ alkaline phosphatase, $B U N$ blood urea nitrogen, $C E R A$ continuous erythropoietin receptor activator, $C R P$ $C$-reactive protein, $d B P$ diastolic blood pressure, $d B P$ pre-HD diastolic blood pressure pre-hemodialysis, ESRD end-stage renal disease, $H D$ hemodialysis, $i P T H$ intact parathyroid hormone, NIHSS National Institute of Health Stroke Scale, $s B P$ systolic blood pressure, $s B P$ pre-HD systolic blood pressure pre-hemodialysis, $T$-Chol total cholesterol, TSAT transferrin saturation

Continuous variables are shown as mean \pm standard deviation and categorical variables, as percentage or number (percentage)

${ }^{a}$ Median (interquartile range)

replacement were independently associated with the severity of cerebral hemorrhage. The corrected serum calcium had the greatest effect on cerebral hemorrhage among them $(P<0.001)$ (Table 3$)$.

\section{Differences between cerebral hemorrhage group and control group}

As shown in Table 2 (right side), significant differences were noted in the dialyzing time, BP pre-HD, proportion of online hemodiafiltration (HDF), erythropoiesis-stimulating agent (ESA) doses, ferritin levels, blood urea nitrogen (BUN) levels, corrected serum calcium levels, serum phosphate levels, and iPTH between cerebral hemorrhage group and control group.

The univariate logistic regression model was applied to these factors and the predetermined traditional risk factors, and multivariable logistic regression analysis was performed subsequently. Model 1 was composed of risk factors for traditional cerebral hemorrhage, and model 2 was created by stepwise methods. Although sBP pre-HD had the greatest association with cerebral hemorrhage in both models, the corrected serum calcium and iPTH were also significant parameters for cerebral hemorrhage (Table 4).

\section{Predictors of hematoma volume}

To determine the predictors and identify the relationship between the corrected serum calcium level and hematoma volume, multiple regression analysis was performed. Model 1 consisted of predetermined factors, and model 2 included use of antiplatelet in addition to the model 1 factors. Multiple regression analysis indicated that the size of the hematoma was significantly correlated with the corrected serum calcium level ( $\beta$ 0.378; standard error (SE) 0.101; $\mathrm{P}<0.001$ ) and antiplatelet use ( $\beta$ 0.378; SE 0.089; $P=0.005$ ) (model 2; $\mathrm{R}^{2}=0.26$ ); however, sBP pre-HD proximate to the onset had no significant correlation with hematoma volume $(P=0.09)$ (Table 5).

Neurological prognosis and corrected serum calcium level To elucidate the corrected serum calcium levels and neurological prognoses of cerebral hemorrhage, the patients with cerebral hemorrhage were divided into four groups according to the corrected serum calcium levels (Q1-Q4): Q1 (corrected serum calcium $\leq 8.8 \mathrm{mg} / \mathrm{dL}$ ), Q2 (8.9-9.5 mg/dL), Q3 (9.6-10.1 mg/dL), and Q4 ( $\geq 10.2$ $\mathrm{mg} / \mathrm{dL}$ ), and the severity of ADL (mRS) was analyzed. The proportions of patients with severe cerebral hemorrhage, who were mRS5 (severe disabled, bed ridden) and mRS 6 (in-hospital death) were as follows: Q1, 57\%; Q2, 63\%; Q3, 67\%; and Q4, 83\% (Fig. 3). In addition to patients who died and those who survived, the severity of ADL (mRS) at discharge was also significantly associated with high calcium levels $(P=0.02$ by CochranArmitage test).

\section{Correlation between serum calcium levels and factors that could affect serum calcium levels in patients with cerebral hemorrhage}

We attempted to elucidate factors that affected the serum calcium levels in patients with cerebral hemorrhage as follows: serum phosphate level, iPTH level, serum alkaline phosphatase (ALP) level, vitamin D, cinacalcet, calcium bicarbonate, dialysate calcium level, and before onset ADLs (cut-off point was $\mathrm{mRS} 3 ; \mathrm{mRS} \leq 3$ could walk independently), which were shown by the quartiles of the corrected 


\section{a Location of hemorrhage}

Numbers of patients

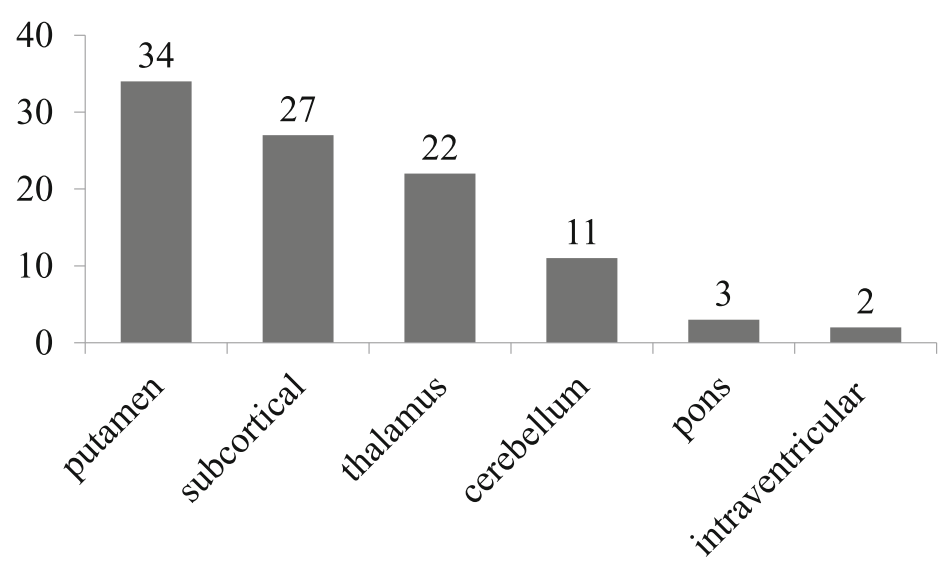

\section{b Histogram for hematoma volume}

Numbers of patients

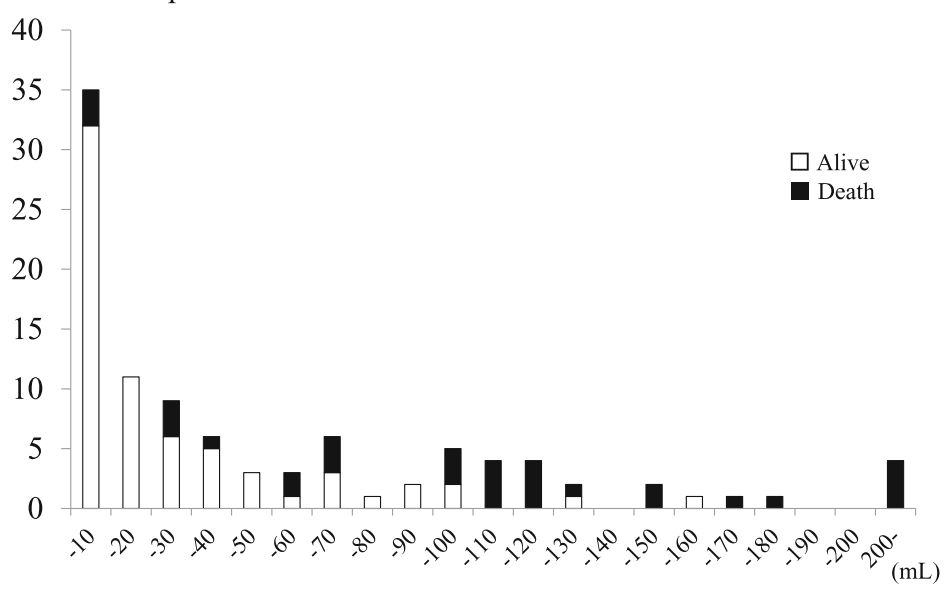

c
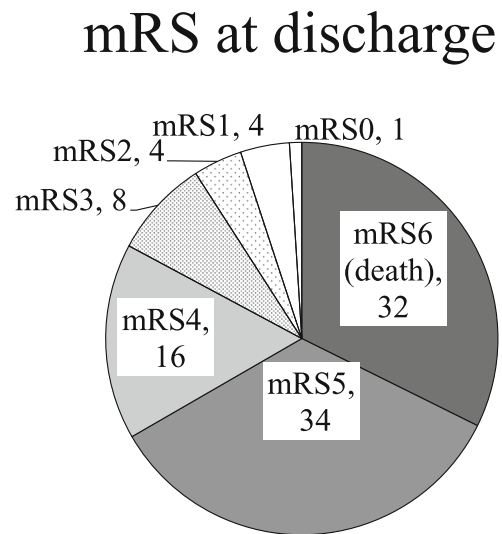

Fig. 2 Characteristics of patients with hematoma. a Location of hematoma. b Distribution of hematoma volume. c Neurological severity outcomes, as a severity of cerebral hemorrhage. The numbers in these figures represent the numbers of patients 
Table 3 Univariate and multivariate logistic regression analyses for prognosis

\begin{tabular}{|c|c|c|c|c|c|c|c|c|c|}
\hline & \multicolumn{3}{|c|}{ Univariate } & \multicolumn{3}{|c|}{ Model 1} & \multicolumn{3}{|c|}{ Model 2} \\
\hline & $\mathrm{OR}$ & $95 \% \mathrm{Cl}$ & $P$ value & $\mathrm{OR}$ & $95 \% \mathrm{Cl}$ & $P$ value & $\mathrm{OR}$ & $95 \% \mathrm{Cl}$ & $P$ value \\
\hline Age per year & 1.00 & $0.96-1.04$ & 0.9 & 1.00 & $0.96-1.05$ & 0.9 & & & \\
\hline Male vs. female & 0.76 & $0.31-1.84$ & 0.5 & 0.88 & $0.34-2.32$ & 0.8 & & & \\
\hline Dialysis vintage & 1.00 & $0.95-1.07$ & 0.8 & & & & & & \\
\hline History of diabetes & 1.33 & $0.56-3.14$ & 0.5 & & & & & & \\
\hline Ischemic heart disease & 5.33 & $1.75-16.2$ & 0.002 & & & & & & \\
\hline Heart valve repair or replacement & 12.0 & $1.34-108$ & 0.03 & & & & 32.4 & $2.61-403$ & 0.007 \\
\hline Antiplatelet use & 3.26 & $1.34-7.93$ & 0.008 & & & & 4.30 & $1.52-12.2$ & 0.006 \\
\hline Warfarin use & 4.13 & $1.23-13.9$ & 0.02 & & & & & & \\
\hline $\mathrm{sBP}$ pre-HD proximate the onset per $10 \mathrm{mmHg}$ & 1.04 & $0.92-1.18$ & 0.5 & & & & & & \\
\hline Corrected serum calcium per mg/dL & 2.38 & $1.39-4.09$ & $<0.001$ & 2.37 & $1.42-4.23$ & $<0.001$ & 2.91 & $1.58-5.35$ & $<0.001$ \\
\hline Phosphate per mg/dL & 1.21 & $0.93-1.58$ & 0.2 & & & & & & \\
\hline iPTH per 10 pg/mL & 1.01 & $0.97-1.05$ & 0.7 & & & & & & \\
\hline Serum albumin per $\mathrm{g} / \mathrm{dL}$ & 0.46 & $0.20-1.05$ & 0.06 & & & & & & \\
\hline
\end{tabular}

Cl confidential interval, $O R$ odds ratio

Model 1: including age, sex, and corrected serum calcium

Model 2: including repair and/or replacement, antiplatelet use, and corrected serum calcium. Valve repair and/or replacement and antiplatelet use were selected by the stepwise method (mixed method)

Table 4 Univariate and multivariate logistic regression analyses for the onset of cerebral hemorrhage

\begin{tabular}{|c|c|c|c|c|c|c|c|c|c|}
\hline & \multicolumn{3}{|c|}{ Univariate } & \multicolumn{3}{|c|}{ Model 1} & \multicolumn{3}{|c|}{ Model 2} \\
\hline & $\overline{\mathrm{OR}}$ & $95 \% \mathrm{Cl}$ & $P$ value & $\overline{\mathrm{OR}}$ & $95 \% \mathrm{Cl}$ & $P$ value & $\overline{\mathrm{OR}}$ & $95 \% \mathrm{Cl}$ & $P$ value \\
\hline Age per year & 0.98 & $0.97-1.00$ & 0.08 & 0.99 & $0.97-1.02$ & 0.5 & & & \\
\hline Male vs. female & 1.49 & $0.93-2.39$ & 0.09 & 1.63 & $0.86-3.11$ & 0.1 & & & \\
\hline HD vintage per year & 1.02 & $0.99-1.05$ & 0.14 & 1.02 & $0.98-1.07$ & 0.3 & & & \\
\hline History of diabetes & 1.22 & $0.77-1.95$ & 0.4 & 1.01 & $0.51-2.01$ & 0.9 & & & \\
\hline Number of antihypertensive drugs & 1.09 & $0.93-1.27$ & 0.3 & & & & & & \\
\hline Warfarin use & 1.98 & $0.97-4.05$ & 0.07 & & & & 1.45 & $0.48-4.41$ & 0.5 \\
\hline Dialyzing time per hour & 1.54 & $1.05-2.23$ & 0.03 & & & & 1.55 & $0.95-2.52$ & 0.08 \\
\hline Online HDF & 0.36 & $0.17-0.74$ & 0.002 & & & & 0.42 & $0.17-1.01$ & 0.052 \\
\hline ESA per $100 \mathrm{U}$ & 1.00 & $1.00-1.01$ & 0.07 & & & & 1.00 & $1.00-1.01$ & 0.5 \\
\hline Intravenous Fe & 1.61 & $0.95-2.70$ & 0.07 & & & & 1.60 & $0.81-3.22$ & 0.2 \\
\hline sBP pre-HD per $10 \mathrm{mmHg}$ & 1.33 & $1.21-1.47$ & $<0.001$ & 1.41 & $1.23-1.61$ & $<0.001$ & 1.40 & $1.23-1.59$ & $<0.001$ \\
\hline $\mathrm{Hb}$ per $\mathrm{g} / \mathrm{dL}$ & 0.88 & $0.75-1.04$ & 0.13 & 0.82 & $0.66-1.02$ & 0.07 & & & \\
\hline Ferritin per $10 \mathrm{ng} / \mathrm{mL}$ & 1.01 & $1.00-1.02$ & 0.2 & & & & & & \\
\hline BUN per $10 \mathrm{mg} / \mathrm{dL}$ & 0.79 & $0.70-0.90$ & $<0.001$ & & & & 0.85 & $0.71-1.01$ & 0.06 \\
\hline Corrected serum calcium per mg/dL & 1.40 & $1.07-1.83$ & 0.01 & 1.52 & $1.05-2.20$ & 0.03 & 1.54 & $1.07-2.22$ & 0.02 \\
\hline Phosphate per mg/dL & 0.82 & $0.70-0.95$ & 0.007 & 0.81 & $0.66-0.98$ & 0.03 & 0.86 & $0.71-1.05$ & 0.1 \\
\hline iPTH per 10 pg/mL & 1.02 & $1.01-1.04$ & 0.02 & 1.03 & $1.00-1.05$ & 0.03 & 1.03 & $1.01-1.05$ & 0.02 \\
\hline T-Chol per $10 \mathrm{mg} / \mathrm{dL}$ & 0.95 & $0.89-1.01$ & 0.12 & & & & & & \\
\hline
\end{tabular}

BUN blood urea nitrogen, $C$ confidential interval, ESA erythropoiesis-stimulating agent, $H D$ hemodialysis, $H D F$ hemodiafiltration, $i P T H$ intact parathyroid hormone, $O R$ odds ratio, $S B P$ pre-HD systolic blood pressure pre-hemodialysis

Model 1: Adjusted by age and sex

Model 2: Adjusted by corrected serum calcium, dialyzing time, online hemodiafiltration, sBP pre-HD, BUN, phosphate, iPTH (parameters were selected by the stepwise method (mixed method)) 
Table 5 Multiple linear regression analysis model for hematoma volume (logarithmic converted)

\begin{tabular}{|c|c|c|c|c|c|c|}
\hline & \multicolumn{3}{|c|}{ Model 1} & \multicolumn{3}{|c|}{ Model 2} \\
\hline & $\beta$ & Standard Error & $P$ value & $\beta$ & Standard Error & $P$ value \\
\hline Age (year) & 0.011 & 0.008 & 0.2 & 0.007 & 0.008 & 0.4 \\
\hline Female & -0.031 & 0.086 & 0.7 & -0.013 & 0.089 & 0.9 \\
\hline sBP pre-HD proximate the onset $(/ \mathrm{mmHg})$ & 0.007 & 0.003 & 0.08 & 0.006 & 0.003 & 0.09 \\
\hline Corrected serum calcium (mg/dL) & 0.381 & 0.093 & 0.02 & 0.378 & 0.101 & $<0.001$ \\
\hline Antiplatelet use & & & & 0.24 & 0.089 & 0.005 \\
\hline$R^{2}$ & & 0.17 & & & 0.26 & \\
\hline
\end{tabular}

dBP pre-HD diastolic pressure pre-hemodialysis, iPTH intact parathyroid hormone, sBP pre-HD systolic blood pressure pre-hemodialysis

Model 1: including age, sex, SBP pre-HD proximate the onset, corrected serum calcium

Model 2: in addition to the model 1's parameter, antiplatelet use was selected by stepwise method (mixed method)

serum levels. However, no significant difference was observed in the serum calcium levels and any of the mentioned factors (Additional file 1 Table 1).

\section{Discussion}

To our knowledge, this is the first study to elucidate the association between serum calcium and cerebral hemorrhage in patients on HD. The corrected serum calcium was positively correlated with the prognosis: disabilities and death from cerebral hemorrhage, hematoma volume, and onset of cerebral hemorrhage in HD patients. After cerebral hemorrhage, more than half of the patients were bedridden or dead (Fig. 3), and the occurrence of cerebral hemorrhage in HD patients should be reduced.
According to previous studies [9, 12], no significant association was found between serum calcium and cerebral hemorrhage; however, data proximate to the cerebral hemorrhage onset were not available in these studies. In our study, serum calcium values were collected close to onset of cerebral hemorrhage and were associated with the prognosis and deterioration of ADL after cerebral hemorrhage (Table 2, Fig. 3). Although precise ADL levels before onset could not be evaluated in the cerebral hemorrhage group, the proportion of walking independently in this group was not very low (Table 2). In this study, the higher the serum calcium level, the lower the ADL after cerebral hemorrhage (Fig. 3).

In the general population, a lower serum calcium level is inversely associated with hematoma volume and poor

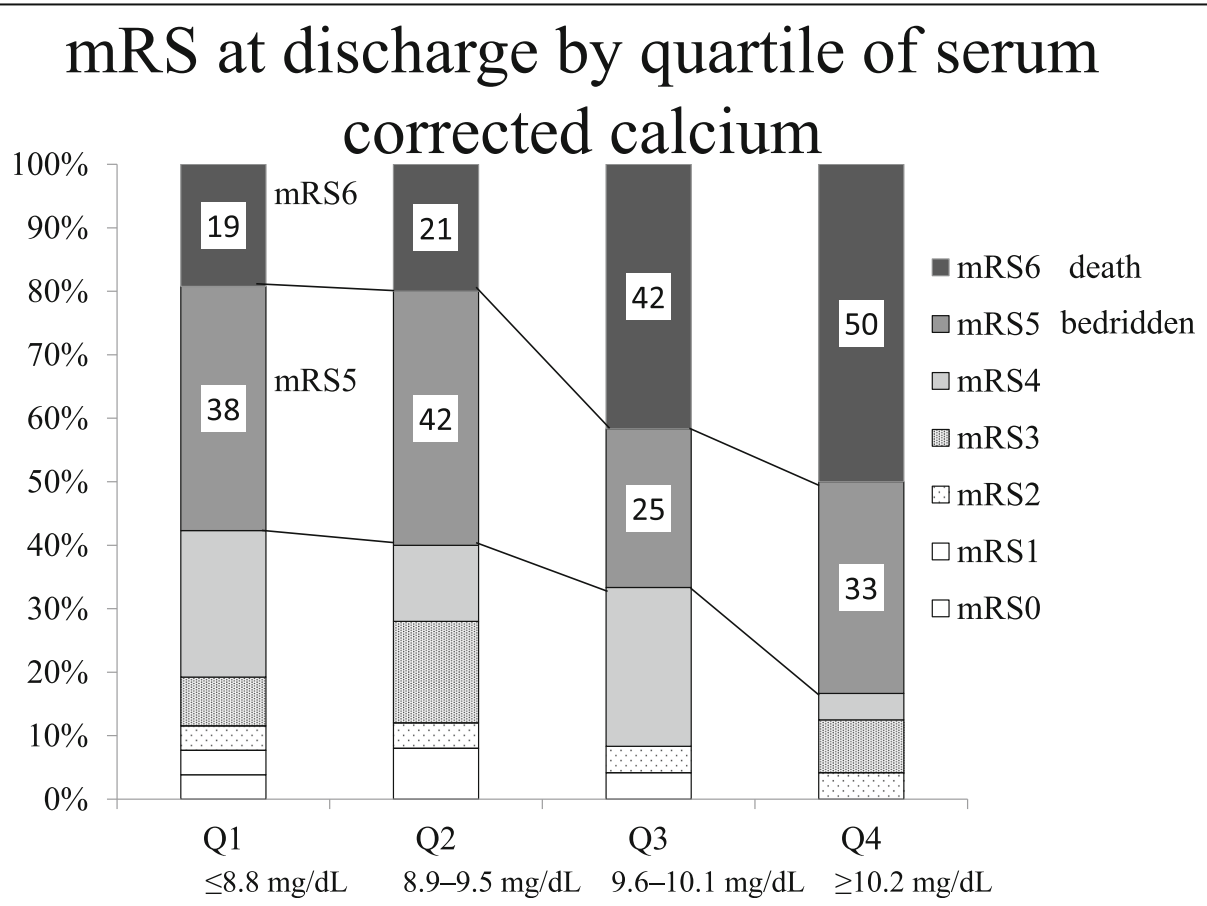

Fig. 3 Distribution on the modified Rankin scale score at discharge by the quartile of the corrected serum calcium. Numbers represent the proportions of patients according to the modified Rankin scale. mRS: modified Rankin Scale 
outcomes [18-20]. In our study, there was no inhospital death in patients whose corrected serum calcium were $<8.4 \mathrm{mg} / \mathrm{dL}$, suggesting that there was no Ushaped or J-shaped phenomenon between the serum calcium and cerebral hemorrhage in HD patients. Although our analyses could not establish the precise relationship between the serum calcium level and cerebral hemorrhage, several possible mechanisms may explain it. First, a high serum calcium level is associated with fragile arteries due to vascular calcification [28]. Second, changes in calcium homeostasis may affect the vascular tone [29] and function of the blood-brain barrier [30]. Calcium may alter the integrity of blood-brain barrier via several molecular mechanisms and direct interaction of calcium ions with junctional proteins [30]. Unlike the general population, the serum calcium level transition during HD should be considered in these patients [31]. Lowering the calcium concentration in the dialysate is associated with cardiovascular instability and arrhythmias [32]; therefore, the use of low calcium concentration dialysate for calcium reduction was not recommended [33]. From these points of view, calcium concentration transition might be associated with cerebral hemorrhage. Third, the possibility that patients with high serum calcium level had low serum albumin and ADL levels cannot be excluded. We could not adjust the preonset ADL levels, which may affect the ADL after cerebral hemorrhage (Fig. 3).

In this study, more than half of patients in the cerebral hemorrhage and control groups were prescribed vitamin D. As vitamin D increases the serum calcium level, it is not advisable to use vitamin $\mathrm{D}$ for secondary hyperparathyroidism routinely [16]. Recently, calcimimetics, such as cinacalcet and etelcalcetide, have been widely used for secondary hyperparathyroidism [34], and they can be considered as the first-choice treatment for secondary hyperparathyroidism instead of vitamin D. However, a low serum calcium level is a risk factor for adverse effect, such as sudden death due to arrhythmia, then serum calcium monitoring is needed.

Based on previous reports in the general population, antiplatelet therapy was proven to be associated with a significantly increased risk of cerebral hemorrhage [35], subsequent poor prognosis [36], and early hematoma growth [36]; a strong association was found between antiplatelet therapy and a poor outcome and hematoma volume in cerebral hemorrhage. Therefore, HD patients prescribed antiplatelets should be monitored for occurrence of bleeding.

This study has some limitations. Its generalizability may be limited by its retrospective study design. The study was conducted in Nagasaki area, which might make generalization of the present results to other regions difficult. The number of outcomes and patients were not enough to adjust for confounding factors. Information bias, especially in past histories of complications, could not be excluded. HD patients with cerebral hemorrhage received treatment at several HD centers; therefore, the treatment policies and modalities differed among facilities, and interlaboratory discrepancies in blood examinations cannot be excluded.

This study has several notable strengths. First, the details of patients with cerebral hemorrhage were available. Data on the parameters were collected just before the onset of cerebral hemorrhage, and data on detailed neurological parameters, such as GCS, NIHSS, mRS, and hematoma volume, were obtained after its onset, unlike that in previous studies. Second, the number of cases in this study was higher than those in previous studies [2, 3, 7, 9-11]. The incidence of cerebral hemorrhage was lower than that of other cardiovascular complications in HD patients, so the numbers of cases in previous studies was limited. Third, most HD patients with cerebral hemorrhage in the Nagasaki City medical zone, except cases of sudden death, were supposed to be included during the observation period. The Nagasaki City medical zone, surrounded by sea and hills, is geographically isolated. Nagasaki City and the neighboring towns, Togitsu and Nagayo, are among the medical care zone areas in Nagasaki prefecture, and their population has been around 500,000 in the last 10 years; however, the population of HD patients has increased slightly from 1297 to 1464 between 2007 and 2016 [37]. Unlike other facilities in the Nagasaki medical zone, Nagasaki University Hospital has a stroke hotline and a HD center; therefore, almost all HD patients with cerebral hemorrhage were transferred to this hospital.

\section{Conclusion}

To prevent the onset and progression of cerebral hemorrhage, serum calcium levels in HD patients should be monitored. Further examinations of the direct relationship between high serum calcium concentrations and cerebral hemorrhage in HD patients are warranted.

\section{Additional file}

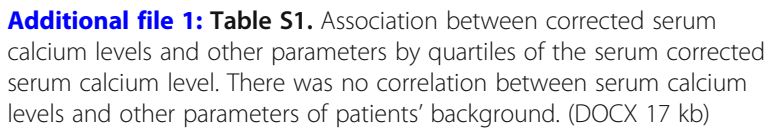

Additional file 1: Table S1. Association between corrected serum calcium levels and other parameters by quartiles of the serum corrected serum calcium level. There was no correlation between serum calcium levels and other parameters of patients' background. (DOCX $17 \mathrm{~kb}$ )

\section{Abbreviations}

ADL: Activities of daily living; ALP: Alkaline phosphatase; BUN: Blood urea nitrogen; CKD: Chronic kidney disease; ESA: Erythropoiesis-stimulating agent; ESRD: End-stage renal disease; GCS: Glasgow Coma Scale; HD: Hemodialysis; HDF: Hemodiafiltration; iPTH: Intact parathyroid hormone; mRS: Modified Rankin scale; NIHSS: National Institutes of Health Stroke Scale; sBP: Systolic blood pressure

\section{Acknowledgments}

We would like to express special thanks to Tomoko Kawaguchi and Koji Ide for creating the databases. 


\section{Authors' contributions}

Research idea and study design: MK, YT, SS, SF, AT, and TN; data acquisition: MK, YT, SK, YO, KM, TU, YM, MN, TI, TH, and SF; data analysis/interpretation: MK, YT, SS; statistical analysis: MK, and SS; supervision or mentorship: YO, TM, AT, HS, $\mathrm{HM}$, and TN. All authors contributed important intellectual content during manuscript drafting. All authors read and approved the final manuscript.

\section{Funding}

This study was supported by a Grant-in-Aid for Scientific Research (KAKENHI; grant number 19 K17747).

\section{Availability of data and materials}

The datasets used and/or analyzed during the current study are available from the corresponding author on reasonable request.

\section{Ethics approval and consent to participate}

This study was approved by the ethics committees of Nagasaki University Hospital (Nagasaki, Japan) (1602221-2) and Nagasaki Renal Center (Nagasaki, Japan) (30001). The ethics committees waived the requirement for written consent due to the retrospective record-based design.

\section{Consent for publication}

Not applicable.

\section{Competing interests}

The authors declare that they have no competing interests.

\section{Author details}

${ }^{1}$ Division of Blood Purification, Nagasaki University Hospital, Nagasaki, Japan 2Department of Nephrology, Nagasaki University Hospital, Nagasaki, Japan. ${ }^{3}$ Department of Neurology and Strokology, Nagasaki University Hospital, Nagasaki, Japan. ${ }^{4}$ Clinical Research Center, Nagasaki University Hospital, Nagasaki, Japan. ${ }^{5}$ Department of Urology, Nagasaki Medical Center, Omura, Japan. ${ }^{6}$ Department of Neurosurgery, Nagasaki University Graduate School of Biomedical Sciences, Nagasaki, Japan. ${ }^{7}$ Department of Nephrology, Nagasaki Renal Center, Nagasaki, Japan. ${ }^{8}$ Department of Respiratory Medicine, Unit of Basic Medical Sciences, Nagasaki University Graduate School of Biomedical Sciences, Nagasaki, Japan.

\section{Received: 23 November 2018 Accepted: 28 May 2019}

Published online: 07 June 2019

\section{References}

1. Drew DA, Sarnak MJ. Ischemic and hemorrhagic stroke: high incidence in hemodialysis and peritoneal dialysis patients. Am J Kidney Dis. National Kidney Foundation, Inc.; 2014:63:547-8.

2. Power A, Chan K, Singh SK, Taube D, Duncan N. Appraising stroke risk in maintenance hemodialysis patients: a large single-center cohort study. Am J Kidney Dis. Elsevier Inc.; 2012;59:249-57.

3. Kawamura M, Fijimoto S, Hisanaga S, Yamamoto Y, Eto T. Incidence, outcome, and risk factors of cerebrovascular events in patients undergoing maintenance hemodialysis. Am J Kidney Dis. 1998:31:991-6.

4. Herrington W, Haynes R, Staplin N, Emberson J, Baigent C, Landray M. Evidence for the prevention and treatment of stroke in dialysis patients. Semin Dial. 2015;28:35-47.

5. Iseki K, Kinjo K, Kimura Y, Osawa A, Fukiyama K. Evidence for high risk of cerebral hemorrhage in chronic dialysis patients. Kidney Int. 1993:44:1086-90.

6. Wakasugi M, Matsuo K, Kazama JJ, Narita I. Higher mortality due to intracerebral hemorrhage in dialysis patients: a comparison with the general population in Japan. Ther Apher Dial. 2015;19:45-9.

7. Toyoda K, Fujii K, Fujimi S, Kumai Y, Tsuchimochi H, Ibayashi S, et al. Stroke in patients on maintenance hemodialysis: a 22-year single-center study. Am J Kidney Dis. 2005;45:1058-66.

8. Wang HH, Hung SY, Sung JM, Hung KY, Wang JD. Risk of stroke in longterm dialysis patients compared with the general population. Am J Kidney Dis. 2014;63:604-11.

9. Yamada S, Tsuruya K, Taniguchi M, Tokumoto M, Fujisaki K, Hirakata H, et al. Association between serum phosphate levels and stroke risk in patients undergoing hemodialysis: the Q-cohort study. Stroke. 2016;47:2189-96.
10. Sakamoto N, Ishikawa E, Aoki K, Uemae Y, Komatsu Y, Matsumura A. Clinical outcomes of intracerebral hemorrhage in hemodialysis patients. World Neurosurg Elsevier Inc. 2014;81:538-42.

11. Yotsueda R, Tanaka S, Taniguchi M, Fujisaki K, Torisu K, Masutani K, et al. Hemoglobin concentration and the risk of hemorrhagic and ischemic stroke in patients undergoing hemodialysis: the Q-cohort study. Nephrol Dial Transplant. 2017:1-9.

12. Tagawa M, Hamano T, Nishi H, Tsuchida K, Hanafusa N, Fukatsu A, et al. Mineral metabolism markers are associated with myocardial infarction and hemorrhagic stroke but not ischemic stroke in hemodialysis patients: a longitudinal study. PLoS One. 2014;9:1-16.

13. Spiegel DM, Brady K. Calcium balance in normal individuals and in patients with chronic kidney disease on low- and high-calcium diets. Kidney Int. 2012:81:1116-22

14. Nakano C, Hamano T, Fujii N, Matsui I, Tomida K, Mikami S, et al. Combined use of vitamin D status and FGF23 for risk stratification of renal outcome. Clin J Am Soc Nephrol. 2012;7:810-9.

15. Tentori F, Blayney MJ, Albert JM, Gillespie BW, Kerr PG, Bommer J, et al. Mortality risk for dialysis patients with different levels of serum calcium, phosphorus, and PTH: the Dialysis outcomes and practice patterns study (DOPPS). Am J Kidney Dis. 2008;52:519-30.

16. Moe SM, Drueke TB. Group for the KW. KDIGO clinical practice guideline for the diagnosis, evaluation, prevention and treatment of chronic kidney disease mineral and bone disorder (CKD-MBD). Kidney Int. 2017;76:S1-128.

17. Ketteler M, Block GA, Evenepoel P, Fukagawa M, Herzog CA, McCann L, et al. Executive summary of the $2017 \mathrm{KDIGO}$ chronic kidney disease-mineral and bone disorder (CKD-MBD) guideline update: what's changed and why it matters. Kidney Int. 2017;92:26-36.

18. Inoue $Y$, Miyashita F, Toyoda K, Minematsu K. Low serum calcium levels contribute to larger hematoma volume in acute intracerebral hemorrhage. Stroke. 2013:44:2004-6.

19. You S, Han Q, Xu J, Zhong C, Zhang YY, Liu H, et al. Serum calcium and phosphate levels and short- and long-term outcomes in acute intracerebral hemorrhage patients. J Stroke Cerebrovasc Dis. 2016;25:914-20.

20. Morotti A, Charidimou A, Phuah C-L, Jessel MJ, Schwab K, Ayres AM, et al. Association between serum calcium level and extent of bleeding in patients with intracerebral hemorrhage. JAMA Neurol. 2016;73:1285.

21. Helweg-Larsen S, Sommer W, Strange P, Lester J, Boysen G. Prognosis for patients treated conservatively for spontaneous intracerebral hematomas. Stroke. 1984;15:1045-8.

22. Falcone GJ, Biffi A, Brouwers HB, Anderson CD, Battey TWK, Ayres AM, et al. Predictors of hematoma volume in deep and lobar supratentorial intracerebral hemorrhage. JAMA Neurol. 2013;70:988-94.

23. Broderick JP, Brott TG, Duldner JE, Tomsick T, Huster G. Volume of intracerebral hemorrhage a powerful and easy-to-use predictor of 30-day mortality. 1993:987-93.

24. Brott T, Jr HPA, Olinger CP, Marler JR, Barsan WG. Biller J, et al. Measurements of Acute cerebral infarction. 2016:1:864-71.

25. Sulter G, Steen C, De Keyser J. Use of the Barthel index and modified Rankin scale in acute stroke trials. Stroke. 1999:30:1538-41.

26. Payne RB, Little AJ, Williams RB, Milner JR. Interpretation of serum calcium in patients with abnormal serum proteins. Br Med J. 1973:4:643-6.

27. Yoshida T, Hayashi M. Anemia treatment by erythropoiesis-stimulating agents during the 6 months before the initiation of hemodialysis: comparison of darbepoetin alfa and continuous erythropoietin receptor activator. Keio J Med. 2017;66:44-50.

28. O'Neill WC. Targeting serum calcium in chronic kidney disease and end stage renal disease: is normal too high? Kidney Int. 2016;89:40-5.

29. Marín J, Encabo A, Briones A, García-Cohen EC, Alonso MJ. Mechanisms involved in the cellular calcium homeostasis in vascular smooth muscle: Calcium pumps Life Sci 1999;64:279-303.

30. Brown RC, Davis TP. Calcium modulation of adherens and tight junction function: a potential mechanism for blood-brain barrier disruption after stroke. Stroke. 2002;33:1706-11

31. Kyriazis J, Glotsos J, Bilirakis L, Smirnioudis N, Tripolitou M, Georgiakodis F, et al. Dialysate calcium profiling during hemodialysis: use and clinical implications. Kidney Int. 2002;61:276-87.

32. Drüeke TB, Touam M. Calcium balance in haemodialysis - do not lower the dialysate calcium concentration too much (con part). Nephrol Dial Transplant. 2009;24:2990-3.

33. Isakova T, Nickolas TL, Denburg M, Yarlagadda S, Weiner DE, Gutiérrez OM, et al. KDOQI US commentary on the 2017 KDIGO clinical practice guideline 
update for the diagnosis, evaluation, prevention, and treatment of chronic kidney disease-mineral and bone disorder (CKD-MBD). Am J Kidney Dis. 2017;70:737-51.

34. Pereira L, Meng C, Marques D, Frazão JM. Old and new calcimimetics for treatment of secondary hyperparathyroidism: impact on biochemical and relevant clinical outcomes. Clin Kidney J. 2018;11:80-8.

35. He J, Whelton PK, Vu B, Klag MJ. Aspirin and risk of hemorrhagic stroke: a meta-analysis of randomized controlled trials. J Am Med Assoc. 1998;280: 1930-5.

36. Naidech AM, Jovanovic B, Liebling S, Garg RK, Bassin SL, Bendok BR, et al. Reduced platelet activity is associated with early clot growth and worse 3 month outcome after intracerebral hemorrhage. Stroke. 2009;40:2398-401.

37. The Nagasaki Regional Council for Renal Failure. Annual data reports of the patients on renal replacement therapy in Nagasaki Prefecture. 2007-2016, not available on web site. p. not available.

\section{Publisher's Note}

Springer Nature remains neutral with regard to jurisdictional claims in published maps and institutional affiliations.

Ready to submit your research? Choose BMC and benefit from:

- fast, convenient online submission

- thorough peer review by experienced researchers in your field

- rapid publication on acceptance

- support for research data, including large and complex data types

- gold Open Access which fosters wider collaboration and increased citations

- maximum visibility for your research: over $100 \mathrm{M}$ website views per year

At BMC, research is always in progress.

Learn more biomedcentral.com/submissions 